\title{
Quelques faits saillants de la conférence Neutrino 2012
}

François Vannucci (vannucci@in2p3.fr)

Laboratoire de physique nucléaire et des hautes énergies, 4 place Jussieu, 75252 Paris Cedex 05

Comme tous les deux ans,

la communauté des physiciens

des neutrinos s'est réunie

pendant une semaine,

lors de la «Conférence

internationale sur la physique

et I'astrophysique des

neutrinos », tenue au début

du mois de juin à Kyoto,

l'ancienne capitale impériale

du Japon. Cette conférence

a réuni 630 physiciens, battant

le record établi par Paris

en 2004 avec 540 participants.

[1] F. Vannucci, Reflets de la physique 27 (décembre 2011 -janvier 2012) 18-19.

(1) www.laguna-science.eu/

\section{Quoi de neuf?}

L'objet d'une conférence est toujours de faire le point sur les résultats expérimentaux récents, discuter les nouvelles idées théoriques en vogue et permettre aux physiciens de la spécialité de se rencontrer pour commenter l'actualité et envisager les axes futurs de recherche.

Ce fut globalement une conférence de très bon cru. À dire vrai, aucune annonce inattendue ne suscita un enthousiasme palpable - internet est plus rapide - mais les nombreux résultats présentés ont permis de préciser de manière décisive le tableau d'ensemble qu'on a des neutrinos.

La conférence débuta par un exposé de J. Steinberger, Prix Nobel 1988 pour la découverte du deuxième type de neutrinos, celui associé au muon. L'année présente marque le cinquantenaire de l'expérience honorée, et il est bon de se pencher sur les grands événements qui ont jalonné l'histoire. L'orateur rappela quelques expériences fondatrices du temps où les mesures se satisfaisaient d'un appareillage tenant sur une paillasse de laboratoire ; la préhistoire avait ses vertus.

Comme dans toute conférence, il existe des sujets " chauds " et d'autres qui le sont moins. La vitesse des neutrinos, qui fit tant de bruit dans les médias à l'automne dernier [1], appartenait évidemment à la première catégorie, même si tous les participants connaissaient à l'avance la conclusion de cet intermède peu glorieux. Quatre expériences différentes ont clos la parenthèse : les neutrinos ne sont pas plus rapides que la lumière, la théorie de la relativité est sauve. La collaboration OPERA, qui mit le feu aux poudres, présenta de nouvelles données obtenues avec un faisceau de neutrinos aux caractéristiques optimisées venant du CERN ; elle réévalua également la vitesse déduite des données précédentes, après avoir identifié une malheureuse erreur de connexion au niveau d'un instrument de mesure. Le résultat final ne révèle aucune déviation par rapport à la vitesse de la lumière. Trois autres expériences, voisines d'OPERA dans le tunnel du Gran Sasso, ont enfoncé le clou : Borexino, Icarus et LVD ont toutes mesuré une vitesse en accord avec l'orthodoxie. Quel enseignement doit-on tirer de cet irritant épisode? Les neutrinos ont bien sûr profité d'une audience inespérée, et cela est positif, mais cet avantage fut obtenu par une sorte d'effraction. L'éthique a été respectée dans la procédure d'analyse des données et on comprend que devant un résultat qui aurait annoncé une révolution intellectuelle de première grandeur, on se sente comme la poule devant le couteau. Pourtant, il reste pour la communauté un parfum d'honneur perdu. On peut s'étonner d'un retentissement planétaire qui ne se serait pas propagé sans l'assentiment de certains. La communication a manqué de prudence. Malgré tout, l'anomalie n'aura duré que six mois, ce qui est fort peu à l'aune des temps caractéristiques que réclament les expériences neutrinos. Dont acte.

\section{L'angle $\theta_{13}$, enfin !}

Mais le résultat de physique le plus important vint de la discussion du dernier angle de mélange entre neutrinos. Les états propres des interactions, $v_{\mathrm{e}}, v_{\mu}$ et $v_{\tau}$, different des états propres de propagation appelés $v_{1}, v_{2}$ et $v_{3}$, et ceci donne lieu au phénomène d'oscillations, c'est-à-dire la mutation spontanée d'un type de neutrino en un autre. Une matrice de mélange $3 \times 3$, fondée sur trois angles, relie les deux 


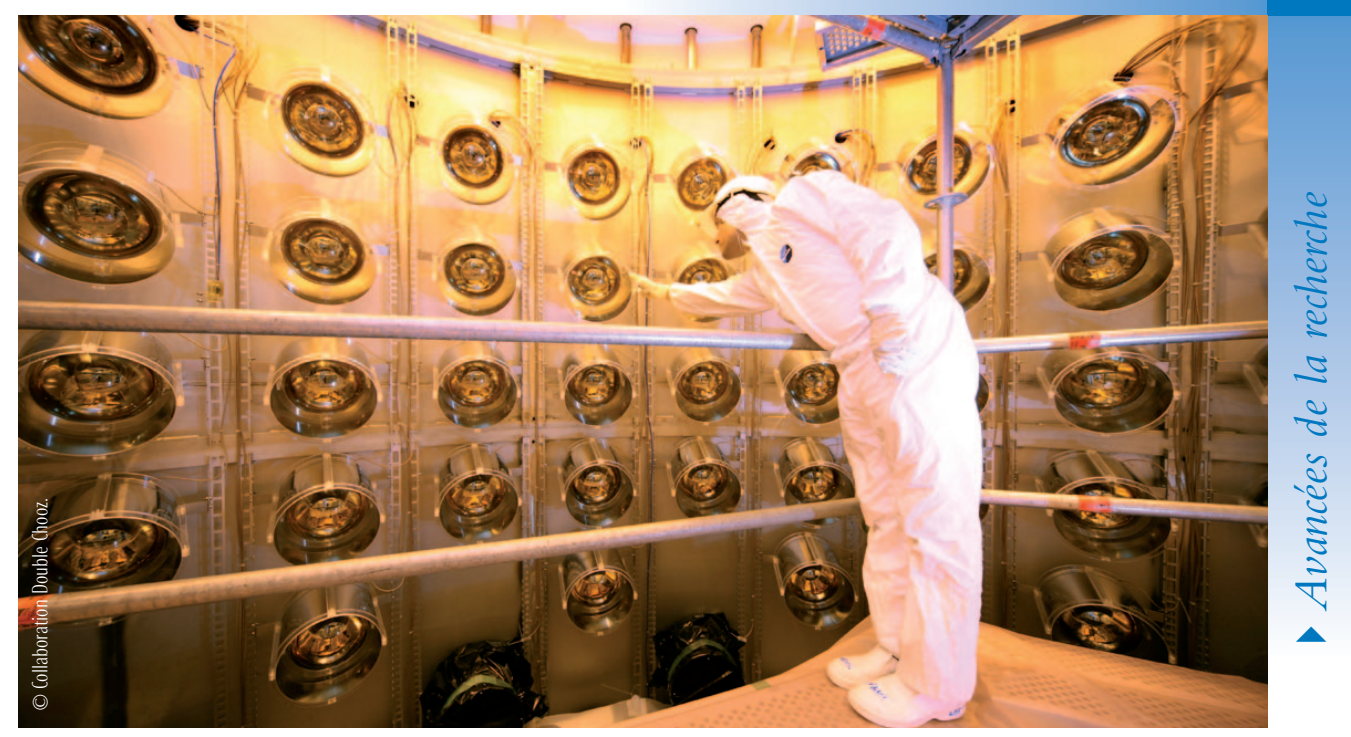

1. Le détecteur Double Chooz, en phase de mise au point.

ensembles d'états. Deux de ces angles sont connus : $\theta_{12}$ grâce aux neutrinos du Soleil, et $\theta_{23}$ grâce à ceux produits par les rayons cosmiques dans l'atmosphère. Le troisième angle, $\theta_{13}$, restait inconnu. En un beau tir groupé, trois expériences montées auprès de réacteurs nucléaires ont présenté des résultats concordants mesurant la disparition de neutrinos en fonction de la distance à la source. La première à dégainer fut Double Chooz, située dans les Ardennes françaises (fig. 1). L'annonce fut dévoilée dès l'automne 2011. Puis l'expérience chinoise Daya Bay suivie de près par l'expérience coréenne RENO, fournirent des résultats plus précis durant le printemps. Notons qu'une première indication vint à l'été 2011 d'une expérience japonaise $\mathrm{T} 2 \mathrm{~K}$, mesurant de manière complémentaire l'apparition de $\boldsymbol{v}_{\mathrm{e}}$ dans un faisceau de $v_{\mu}$. Les mesures s'avèrent en accord et indiquent un angle de mélange d'environ 9 degrés, ce qui fait pousser un ouf ! de soulagement pour la suite des opérations. Aucune prédiction ne fixe cet angle, qui aurait pu se révéler beaucoup plus petit et inaccessible aux expériences en cours. Ceci aurait nécessité un nouveau round d'appareillages plus ambitieux.

Ce résultat est important, du fait qu'il ouvre la voie pour attaquer la violation de CP dans le secteur des leptons. CP associe la symétrie $C$ faisant passer d'une particule à son antiparticule et la symétrie de parité $\mathrm{P}$, image dans un miroir. Ce phénomène, qu'on espère détecter par des probabilités d'oscillations différentes entre neutrinos d'une part et antineutrinos d'autre part, peut sembler technique, mais il est invoqué pour comprendre la disparition de l'antimatière dans l'évolution de l'Univers. C'est donc un sujet d'importance majeure dans la liste des énigmes encore à résoudre.
Un autre sujet " chaud " est celui de l'anomalie constatée dans le flux mesuré de neutrinos de réacteurs (et de sources radioactives) par rapport aux calculs. D. Lhuillier (Irfu-Saclay) présenta une réévaluation de nombreux résultats passés. Une étude très minutieuse montre que, depuis les années 1980, beaucoup d'indications pointent vers un déficit systématique des flux observés par rapport aux prédictions. Au final, 7\% des neutrinos semblent disparaitre entre les points de production et de détection. Ceci pourrait indiquer un effet nucléaire caché, mais une hypothèse plus osée propose l'existence de nouveaux neutrinos, comme discuté par T. Lasserre (Irfu-Saclay et APC, Paris 7). On les appelle " neutrinos stériles ", parce qu'ils ne participent pas aux interactions faibles et ne se couplent aux particules connues que par mélange. En fait, la théorie prédit de tels états, mais on les attend dans un domaine de masses très élevées pratiquement inaccessible ; or, ici, il s'agit de masses permettant une recherche expérimentale rapide. Plusieurs projets sont en discussion pour clarifier le problème.

\section{L'astronomie des neutrinos}

Un autre résultat potentiellement de grand retentissement fut présenté. Un détecteur géant nommé IceCube, consistant en un kilomètre cube de glace instrumenté au Pôle Sud, a montré les premiers signaux de neutrinos de très haute énergie. Jusqu'à présent, IceCube et son concurrent Antarès construit au fond de la Méditerranée près de Toulon n'avaient détecté que des neutrinos attribuables à une production dans l'atmosphère. Celle-ci est effectivement la contribution majeure jusqu'à des énergies de $10^{5} \mathrm{TeV}$, dépassant d'un facteur 10000 celles obtenues par le collisionneur LHC. Les deux événements enregistrés à une énergie encore plus élevée indiqueraient une origine extraterrestre, ce qui pourrait ouvrir l'étude d'objets astrophysiques à l'aide des neutrinos.

\section{Perspectives}

Quand la communauté se retrouve, elle ne manque pas de parler des perspectives. C'est l'avantage du programme de recherche en neutrinos : avoir une feuille de route bien balisée. Maintenant que $\theta_{13}$ est mesuré, il s'agit en premier lieu de fixer l'échelle absolue des trois masses de neutrinos et leur ordonnancement ; puis on s'attaquera à la violation de $\mathrm{CP}$, ce qui constitue le Graal de la discipline. Il est assez facile d'imaginer les expériences nécessaires. Elles seront encore plus ambitieuses que les présentes, mais leurs tailles restent dans le domaine du réalisable. On parle de détecteurs atteignant 100 kilotonnes ou une mégatonne. Ainsi, l'Europe se fédère autour d'un programme appelé LAGUNA ${ }^{(1)}$, qui propose d'envoyer des neutrinos du CERN vers une mine finlandaise à $2300 \mathrm{~km}$ de distance. Le projet se chiffre en milliards d'euros, les physiciens des neutrinos ont du travail pour les 20 années à venir !

$\mathrm{Au}$ sortir de cette conférence, on en sait donc plus sur les neutrinos, et rendez-vous est pris pour la prochaine édition, en juin 2014 à Boston. Ce sera une étape supplémentaire sur le chemin de Zurich, déjà proposé comme site de la rencontre en 2030 pour célébrer le centenaire de la fameuse lettre de W. Pauli suggérant l'existence d'une particule invisible, que trois ans plus tard E. Fermi baptisa du joli nom de neutrino. 